Pacific Journal of Mathematics

OPERATORS WITH FINITE ASCENT 


\title{
OPERATORS WITH FINITE ASCENT
}

\author{
K. B. LAURSEN ${ }^{1}$
}

A continuous linear operator $T$ on a Banach space $X$ is said to have finite ascent if each operator $T-\lambda, \lambda \in \mathbf{C}$, has stabilizing kernel, i.e. for some $n(n$ may depend on $\lambda) \operatorname{ker}(T-\lambda)^{n}=$ $\operatorname{ker}(T-\lambda)^{n+1}$. Easy examples are provided by normal operators in Hilbert space. For operators with finite ascent a description of the maximal algebraic subspaces (defined below) is readily obtained, thus opening up the possibility of automatic continuity theory involving these operators and their intertwiners. This paper makes a beginning. We see that operators with finite ascent have the single valued extension property. We also characterize some of the analytic spectral subspaces, but the main results are obtained for certain subclasses of operators with finite ascent. Three such classes are considered: a class enjoying a polynomial growth condition, very recently introduced by Barnes, the dominant operators in Hilbert space, studied by many authors, and, mostly, the totally paranormal operators, introduced in $\S 4$. A main result is that if $T$ belongs to this class and if $X_{T}(F)$ denotes the analytic spectral subspace with respect to the closed subset $F \subseteq \mathrm{C}$ then $X_{T}(F)$ is closed (Proposition 4.14). If we restrict ourselves to Hilbert space and to such a $T$ without eigenvalues then the algebraic and the analytic spectral subspaces coincide (Proposition 4.15). This result allows us to tap into the already existing fund of automatic continuity theory; the paper includes a sample, intended to illustrate the possibilities.

\section{General and introductory material.}

Definition 1.1. If $T$ is a linear operator on the vector space $X$, then for a not necessarily closed, but $T$-invariant subspace $Z \subseteq X$ we define the spectrum of $T \mid Z$ as the set of complex numbers $\lambda$ for which $(T-\lambda) \mid Z$ is not an isomorphism, i.e. is either not surjective or not injective.

Definition 1.2. If $A \subseteq \mathbf{C}$ then the maximal algebraic spectral subspace $E_{T}(A)$ is the largest subspace of $X$ on which all the restrictions of $T-\lambda, \lambda \in \mathbf{C} \backslash A$, are surjective.

${ }^{1}$ Some of this material was presented to the Workshop on Banach algebras and Banach spaces of continuous functions held at Brautarholt, Iceland, July 24-29, 1989. I wish to express my thanks for all the hospitality extended to the participants in this workshop by the organizer, E. Briem. I would also like to thank Michael M. Neumann for some helpful comments on the material in this paper. 
These spaces and their importance in automatic continuity have been explored in many papers (cf. [8] and [9], where also more references may be found).

One basic property of the $E_{T}$ spaces is that of absorbency. A subspace $Z$ of $X$ is said to be absorbent (with respect to a given subset $B$ of $\mathbf{C}$ ) if for every $\lambda \in B,(T-\lambda) x \in Z$ implies that $x \in Z$. It is shown in [8, Proposition 2.2] that $E_{T}(A)$ is absorbent with respect to $A$. This is usually described with no explicit mention of $A$. Absorbency is used in the proof of the next result.

Proposition 1.3. For any bounded linear operator $T$ on a Banach space $X \quad \sigma\left(T \mid E_{T}(A)\right) \subseteq \sigma\left(T \mid E_{T}(A)^{-}\right) \subseteq \sigma(T)$.

Proof. For the inclusion on the left let $A \subseteq \mathrm{C}$ be given and let $Y:=E_{T}(A)^{-}$be the norm closure of $E_{T}(A)$. Let $\lambda \in \rho(T \mid Y)$; then $T-\lambda$ is $1-1$ on $E_{T}(A)$. Let $y \in E_{T}(A)$ and let $z \in Y$ be chosen so that $(T-\lambda) z=y$. If $\lambda \in A$ then by absorbency $z \in E_{T}(A)$. If $\lambda \in \mathbf{C} \backslash A$ then $z \in E_{T}(A)$ (there is a preimage of $y$ in $E_{T}(A)$, and since $\lambda \in \rho(T \mid Y)$ there is only one in $Y$, so it must be $z)$. We have shown that if $\lambda \in \rho(T \mid Y)$, then $T-\lambda$ is $1-1$ and onto $E_{T}(A)$. Thus $\rho(T \mid Y) \subseteq \mathbf{C} \backslash \sigma\left(T \mid E_{T}(A)\right)$, i.e. $\sigma\left(T \mid E_{T}(A)\right) \subseteq \sigma(T \mid Y)$. The inclusion on the right is [8, Corollary, p. 159].

Definition 1.4. We say that the operator $T$ has finite ascent if for every $\lambda \in \mathbf{C}$ there is an $n \in \mathbf{N}$ such that $\operatorname{ker}(T-\lambda)^{n}=\operatorname{ker}(T-\lambda)^{n+1}$.

In [9] it is shown that generalized scalar operators have finite ascent. Evidently, this property is inherited by any restriction of an operator, so also subscalar operators, in particular hyponormal operators [12], fall in this class. More examples will be given below.

Proposition 1.5. If $T$ has finite ascent then $\sigma\left(T \mid E_{T}(A)\right) \subseteq A$ for any $A \subseteq \mathrm{C}$.

Proof. Let $\lambda \notin A$ and suppose $\operatorname{ker}(T-\lambda)^{n}=\operatorname{ker}(T-\lambda)^{n+1}$. By definition of $E_{T}(A)$, if $x \in E_{T}(A)$ then there is $z \in E_{T}(A)$ for which $x=(T-\lambda)^{n} z$. If $(T-\lambda) x=0$ then $z \in \operatorname{ker}(T-\lambda)^{n+1}=\operatorname{ker}(T-\lambda)^{n}$ and hence $x=0$. Since $(T-\lambda) \mid E_{T}(A)$ is surjective (by definition), this completes the proof.

By referring to [9, Lemma, p. 734] it is easy to give an explicit description of the $E_{T}(A)$-spaces: 
Proposition 1.6. If $T$ has finite ascent then

$$
E_{T}(A)=\bigcap_{\lambda \in \mathbf{C} \backslash A, n \in \mathbf{N}}(T-\lambda)^{n} X .
$$

Definition 1.7 [9]. The analytic residuum $S(T)$ is defined as $S(T):=\{\lambda \in \mathbf{C} \mid$ for every neighborhood $N(\lambda)$ of $\lambda$ there is a neighborhood $N^{\prime} \subseteq N(\lambda)$ and a non-zero analytic function $f: N^{\prime} \rightarrow X$ for which $(T-\mu) f(\mu)=0\}$.

If $S(T)=\varnothing$ then $T$ is said to have the single valued extension property, abbreviated SVEP.

Proposition 1.8. Any operator $T$ with finite ascent has SVEP.

Proof. This is a consequence of [9], where it is shown that $S(T) \subseteq$ $\sigma\left(T \mid E_{T}(A)\right)$ for any $A \subseteq \mathbf{C}$. Hence $S(T) \subseteq A$ for any $A$ and so $S(T)=\varnothing$.

EXAMPLE 1.9. This paper will contain examples of several classes of operators which illustrate the properties under examination here. As the first one, we mention briefly a class of operators recently studied by Barnes [2]. This is the class $\mathscr{P}(X)$ of bounded linear operators $T$ on the Banach space $X$, which satisfy a polynomial growth condition:

$$
\exists K, \delta \in \mathbf{R}_{+}:\|\exp (i t T)\| \leq K\left(1+|t|^{\delta}\right) \quad \text { for all } t \in \mathbf{R} .
$$

Barnes shows that any $T \in \mathscr{P}(X)$ has real spectrum [2, condition III, p. 210]. He also gives numerous examples of operators in $\mathscr{P}(X)$, e.g. hermitian operators, nilpotent operators (and more generally algebraic operators with real spectra). The operators in $\mathscr{P}(X)$ have finite ascent [2, Theorem 8], so the theory developed here is applicable. As pointed out to the author by Michael M. Neumann, [4, Theorem 5.4.5] shows that $\mathscr{P}(X)$ coincides with the class of generalized scalar operators with real spectra. Thus the finite ascent is also a consequence of the proof of [9, Corollary, p. 735]. For the operators of $\S 4$ below we can say even more (Proposition 4.7), when the spectrum is real.

We finish this section with a few remarks that apply to Hilbert space.

Every bounded linear operator $T$ on a Hilbert space has an orthogonal decomposition $T=T_{\text {normal }} \oplus T_{\text {pure }}$ (implemented by a restriction to a reducing subspace), where $T_{\text {normal }}$ is a normal operator and $T_{\text {pure }}$ is pure, which means that no restriction of $T_{\text {pure }}$ to a reducing subspace is normal. Either of the two summands may be absent. 
And while we are on the subject of reducing subspaces: The last little result of this section will be used in $\S 4$. We include it here because it is general.

LEMMA 1.10. For any operator $T \in \mathscr{B}(\mathscr{H})$, if $\lambda$ with $|\lambda|=\|T\|$ is an eigenvalue of $T$ then $\operatorname{ker}(T-\lambda)$ is reducing.

Proof. The operator $T / \lambda$ is a contraction with eigenvalue 1 . By [16, Proposition I.3.1] $T(x) / \lambda=x$ if and only if $(T / \lambda)^{*}(x)=x$. Hence $\operatorname{ker}(T-\lambda)=\operatorname{ker}(T-\lambda)^{*}$.

Note that a consequence of this lemma is that if $T$ is pure and if $\|T\|=r(T)$ (= spectral radius) then there are no eigenvalues $\lambda$ for which $|\lambda|=\|T\|$. For certain classes of operators this claim can be strengthened (cf. Remark 3.3b) below).

\section{Operators with SVEP.}

Definition 2.1. If $T$ is a bounded linear operator on the Banach space $X$ and if $x \in X$ then the local spectrum $\sigma_{T}(x)$ is the complement in $\mathbf{C}$ of the local resolvent $\rho_{T}(x):=\{\lambda \in \mathbf{C} \mid$ in some neighborhood of $\lambda$ the equation $(T-\mu) x(\mu)=x$ has an analytic solution $\}$, and for a given set $A \subseteq \mathbf{C}$ the spectral subspace is $X_{T}(A):=\{x \in$ $\left.X \mid \sigma_{T}(x) \subseteq A\right\}$.

For operators with SVEP we can describe the $X_{T}$ spaces over closed discs. We will use the notation $\mathbf{D}_{r}:=\{z \in \mathbf{C}|| z \mid \leq r\}$ and define $X_{r}:=\bigcap_{\rho>r}\left\{x \in X \mid\right.$ there is a constant $K=K(x, \rho) \in \mathbf{R}_{+}:\left\|T^{n} x\right\| \leq$ $\left.K \rho^{n}, n \in \mathbf{N}\right\}$. Note that $X_{r}$ is a linear space.

Proposition 2.2. If $T \in \mathscr{B}(X)$ has SVEP then $X_{T}\left(\mathbf{D}_{r}\right)=X_{r}$.

Proof. Let $x \in X_{r}$. For $\lambda \notin \mathbf{D}_{r} x(\lambda):=\sum_{n=0}^{\infty} T^{n} x / \lambda^{n+1}$ is an analytic function for which $(T-\lambda) x(\lambda)=x$. Thus $X_{r} \subseteq X_{T}\left(\mathbf{D}_{r}\right)$. For the converse we use an argument related to [3, Lemma 1.3.3]; let $x \in X_{T}\left(\mathbf{D}_{r}\right)$, let $\rho>r$ and let $\Gamma$ be a positively oriented circle, centered at 0 and with radius $\rho$. In $\mathbf{C} \backslash \mathbf{D}_{r}$ there is defined a unique analytic function $x(\lambda)$ for which $(T-\lambda) x(\lambda)=x$. It follows that

$$
T^{n} x=-\frac{1}{2 \pi i} \int_{\Gamma} \lambda^{n} x(\lambda) d \lambda
$$

This is a consequence of the Cauchy integral formula: from analyticity we know that the above integral is equal to the integral

$$
\frac{1}{2 \pi i} \int_{|\lambda|=\|T\|+1} \lambda^{n} x(\lambda) d \lambda,
$$


and since in this latter integral $x(\lambda)=(T-\lambda)^{-1} x$, the former formula follows. Hence $\left\|T^{n} x\right\| \leq(2 \pi)^{-1} 2 \pi \rho \rho^{n} M_{\Gamma}$, where $M_{\Gamma}$ is an upper bound for $\|x(\lambda)\|$ on $\Gamma$. From this estimate we get that

$$
\left\|T^{n} x\right\|^{1 / n} \leq\left(\rho M_{\Gamma}\right)^{1 / n} \rho,
$$

and hence, since $\rho>r$ is arbitrary, that $\lim \sup \left\|T^{n} x\right\|^{1 / n} \leq r$. It follows that $x \in X_{r}$.

Another way of describing $X_{T}\left(\mathbf{D}_{r}\right)$ is clear from the above:

COROLlary 2.3. If $T$ has SVEP then

$$
X_{T}\left(\mathbf{D}_{r}\right)=\left\{x \in X \mid \lim \sup \left\|T^{n} x\right\|^{1 / n} \leq r\right\} .
$$

COROLlary 2.4. If $T$ has SVEP then

$$
X_{T}(\{0\})=\left\{x \in X \mid\left\|T^{n} x\right\|^{1 / n} \rightarrow 0\right\} .
$$

This generalizes Lemma 4.4 .4 of [4].

REMARK 2.5. If $T$ has SVEP and $x \in X$ then a "local spectral radius" may be defined. Corollary 2.3 says that $x \in X_{T}\left(\mathbf{D}_{r}\right)$ if and only if lim sup $\left\|T^{n} x\right\|^{1 / n} \leq r$. So, a reasonable definition of local spectral radius $r(x)$ is this lim sup. For a $T$ in the class to be introduced in $\S 4$ we shall see that $r(x) \geq\|T x\|$.

In the next section we shall apply some of these results to a class of operators with finite ascent in Hilbert space.

3. Dominant operators. A bounded linear operator $T$ on a Hilbert space $\mathscr{H}$ is said to be dominant if for every $\lambda \in \mathbf{C}$ there is a constant $M_{\lambda}$ such that

$$
(T-\lambda)(T-\lambda)^{*} \leq M_{\lambda}(T-\lambda)^{*}(T-\lambda) .
$$

It is worth noting that there are other equivalent conditions; one, proved in [6], is this: for every $\lambda \in \mathbf{C}$ there is an operator $W_{\lambda} \in \mathscr{B}(\mathscr{H})$ such that $T-\lambda=(T-\lambda)^{*} W_{\lambda}$; evidently, this implies that $(T-\lambda) \mathscr{H} \subseteq$ $(T-\lambda)^{*} \mathscr{H}$. Another is that $\left\|(T-\lambda)^{*} x\right\| \leq M_{\lambda}^{1 / 2}\|(T-\lambda) x\|$ for all $x \in \mathscr{H}$. See also [15].

REMARK. It is enough to require that the defining inequalities hold for every $\lambda \in \sigma(T)$, since for $\lambda \in \rho(T)$ we have $\left\|(T-\lambda)^{*} x\right\| \leq$ $\left\|(T-\lambda)^{*}(T-\lambda)^{-1}\right\|\|(T-\lambda) x\|$. 
LEMMA 3.1. If $T$ is dominant then $\operatorname{ker}(T-\lambda)=\operatorname{ker}(T-\lambda)^{2}$ for any $\lambda \in \mathbf{C}$, so $T$ has finite ascent.

Proof. Let $\lambda \in \mathbf{C}$; since $(T-\lambda)^{*}=W_{\lambda}^{*}(T-\lambda)$,

$$
\operatorname{ker}(T-\lambda) \subseteq \operatorname{ker}(T-\lambda)^{*} .
$$

Hence, if $x \in \operatorname{ker}(T-\lambda)^{2}$, then $(T-\lambda) x \in \operatorname{ker}(T-\lambda)^{*}$ and, consequently $0=\left\|(T-\lambda)^{*}(T-\lambda) x\right\|\|x\| \geq\left|\left\langle(T-\lambda)^{*}(T-\lambda) x, x\right\rangle\right|=$ $\|(T-\lambda) x\|^{2}$.

LemmA 3.2. If $T$ is dominant and $A \subseteq \mathbf{C}$ then

$$
E_{T}(A)=\bigcap_{\lambda \in \mathbf{C} \backslash A, n \in \mathbf{N}}(T-\lambda)^{n} \mathscr{H} .
$$

Proof. Proposition 1.6.

Remarks 3.3. (a) An example in [13, Remark 4(b), p. 109] shows that the above result cannot be improved to $E_{T}(A)=\bigcap_{\lambda \in \mathbf{C} \backslash A}(T-$ $\lambda)^{k} \mathscr{H}$, at least not with fixed $k=1$. Can a higher, but fixed value of $k$ be used?

(b) If $T$ is dominant then $T_{\text {pure }}$ is dominant (since the domain of $T_{\text {pure }}$ is a reducing subspace for $T$ ). Moreover, $T_{\text {pure }}$ has no eigenvalues (if $\lambda \in \sigma_{p}\left(T_{\text {pure }}\right)$ then the eigenspace $\operatorname{ker}\left(T_{\text {pure }}-\lambda\right)$ is reducing $\left(\operatorname{ker}\left(T_{\text {pure }}-\lambda\right) \subseteq \operatorname{ker}\left(T_{\text {pure }}-\lambda\right)^{*}\right)$ and hence the restriction of $T_{\text {pure }}$ to $\operatorname{ker}\left(T_{\text {pure }}-\lambda\right)$ is normal). Incidentally, this gives an easy proof that a dominant $T$ has SVEP: $T_{\text {normal }}$ does have SVEP, and any operator with empty point spectrum has SVEP. In particular, this is true for $T_{\text {pure }}$.

Questions. (a) Does a dominant operator have Bishop's property $(\beta)$ ? (For a definition of this concept, see for instance [12, p. 386].) It is worth noting that a dominant operator is not necessarily decomposable-there are dominant $T$ for which $T^{*}$ doesn't even have SVEP: if $T$ is an isometry, then $T^{*} T \geq T T^{*}$, so an isometry is even hyponormal. The unilateral right shift on $l^{2}(\mathbf{N})$ is well known to have an adjoint without SVEP.

(b) A dominant operator does not necessarily have $E_{T}(\varnothing)=\{0\}$ (as an example in [13] shows). Thus $E_{T}(\varnothing)=\{0\}$ is necessary for equality of the $\mathscr{H}_{T}$ and the $E_{T}$ spaces. Is this condition sufficient?

As this next result shows, if $y \in E_{T}(F)$ then $\sigma_{T}(y)$ is "mostly" located inside $F$. 
Proposition 3.4. If $T$ is a dominant operator, if $F \subseteq \mathbf{C}$ is closed and $y \in E_{T}(F)$ then $M:=\sigma_{T}(y) \backslash F$ is nowhere dense.

Proof. We begin by noting that if $Y:=E_{T}(F)^{-}$then all eigenvalues of $T \mid Y$ are in $F$ : if $\lambda \in \sigma(T \mid Y) \backslash F$ and if $y \in \operatorname{ker}(T \mid Y-\lambda)$ then $y \in \operatorname{ker}(T-\lambda)^{*} \cap((T-\lambda) \mathscr{H})^{-}=\{0\}$. Thus, if $(T-\lambda) y(\lambda)=y$ for all $\lambda \notin F$, then $y(\lambda)$ is the unique $E_{T}(F)$-valued function for which this equation holds, and, [3, Lemma 1.3.7], $\|y(\lambda)\|$ is a lower semi-continuous function.

We want to show that for $y \in E_{T}(F), M$ is nowhere dense. If $\Delta$ is a closed disc in $M$ then define, for $n=1,2, \ldots$

$$
F_{n}:=\{\lambda \in \Delta \mid\|y(\lambda)\| \leq n\} .
$$

Each set $F_{n}$ is closed, because $\|y(\lambda)\|$ is lower semi-continuous, so by the Baire category theorem there is an $n$ for which $F_{n}$ has interior points. Fix this $n$. Since $y(\lambda)$ is bounded on $F_{n}, y(\lambda)$ is actually weakly continuous $[1$, p. 22$]$. Now we may proceed exactly as in the proof of [3, Th. 1.3.4]; the assumption of hyponormality in that theorem is used only to establish that $(T-\lambda) \mathscr{H} \subseteq(T-\lambda)^{*} \mathscr{H}$, and this inclusion holds for dominant operators as well. The conclusion is that $y(\lambda)$ is an analytic function on all of the given open subset of $F_{n}$. This contradiction establishes the proposition.

4. Totally paranormal operators. An operator $T$ on a Banach space $X$ is said to be paranormal if $\|T x\|^{2} \leq\left\|T^{2} x\right\|\|x\|$ for all $x \in X$. We shall coin the term totally paranormal, abbreviate it TPN and use it about the class of operators $T$ for which $T-\lambda$ is paranormal for every $\lambda \in \mathbf{C}$. As noted in [1, pp. 174-175] the TPN operators form a proper subclass of the paranormal operators.

Recall that in a Hilbert space $\mathscr{H}$ an operator $T$ is said to be hyponormal if $\left\|T^{*} x\right\| \leq\|T x\|$ for every $x \in \mathscr{H}$. Evidently this class contains all normal operators; it also provides us with an easy example of a subclass of the TPN operators.

\section{PROPOSITION 4.1. Every hyponormal operator is TPN.}

Proof. It is easy to see that a hyponormal operator is paranormal: if $\left\|T^{*} x\right\| \leq\|T x\|$ for every $x \in X$, then $\|T x\|^{2}=\left\langle T^{*} T x, x\right\rangle \leq$ $\left\|T^{*} T x\right\|\|x\| \leq\left\|T^{2} x\right\|\|x\|$ for any $x \in X$. And $T$ is hyponormal if and only if $T-\lambda$ is hyponormal. 
LEMMA 4.2. If $T$ is $T P N$ then $\operatorname{ker}(T-\lambda)=\operatorname{ker}(T-\lambda)^{2}$, for every $\lambda \in \mathbf{C}$ so $T$ has finite ascent.

Proof. $\subseteq$ is trivial. $\supseteq$ follows from the definition of TPN.

Corollary 4.3. If $T$ is TPN, then for any $A \subseteq \mathrm{C} E_{T}(A)=$ $\bigcap_{\lambda \notin A, n \in \mathbf{N}}(T-\lambda)^{n} X$.

Proof. Proposition 1.6.

REMARK. The same example which was used to point out that dominant operators are not necessarily decomposable (Question (a) after Remarks 3.3), namely that of a non-unitary isometry, shows that TPN operators do not have to be decomposable. But conceivably, a TPN operator has $(\beta)$. At any rate we have Proposition 4.7 below.

LEMmA 4.4. If $T$ is TPN and $x \in X$ is a unit vector then for $n=$ $1,2, \ldots$

$$
\|(T-\lambda) x\|^{n} \leq\left\|(T-\lambda)^{n} x\right\| \quad \text { for any } \lambda \in \mathbf{C} .
$$

Proof. It is enough to take $\lambda=0$. By [3, Lemma 1.1.2] it suffices to show that $\left\|T^{n} x\right\|^{2} \leq\left\|T^{n+1} x\right\|\left\|T^{n-1} x\right\|$ for $n=1,2, \ldots$. For $n=1$ this is the definition of TPN. And for general $n$ it goes the same way: $\left\|T^{n} x\right\|^{2}=\left\|T\left(T^{n-1} x\right)\right\|^{2} \leq\left\|T^{2}\left(T^{n-1} x\right)\right\|\left\|T^{n-1} x\right\|$.

A similar estimate will appear in Lemma 4.12 as a step towards proving that the $X_{T}(F)$-spaces are closed when $F$ is closed. But before that, we mention in passing some direct (and surely known) consequences of Lemma 4.4.

COROLlaRY 4.5. If $T$ is paranormal, then $\|T\|=r(T)$, where $r(T)$ denotes spectral radius.

Proof. For a unit vector $x,\|T x\|^{n} \leq\left\|T^{n} x\right\| \leq\left\|T^{n}\right\|$, and hence $r(T) \geq\|T x\|$. Thus $\|T\| \leq r(T) \leq\|T\|$.

REMARK. [13] gives an example of a dominant, quasinilpotent operator. By Corollary 4.5 this operator cannot be TPN.

CoROllaRy 4.6. If $T$ is paranormal and $\sigma(T) \subseteq \mathbf{T}$ then $T$ is an invertible isometry. In particular, if $T \in \mathscr{B}(\mathscr{H})$ then $T$ is unitary. 
Proof. Both $T$ and $T^{-1}$ are paranormal and their spectra are subsets of $\mathbf{T}$. Consequently, by Corollary $4.5\|T\|=\left\|T^{-1}\right\|=1$, and so $T$ is an invertible isometry.

REMARK. If $T \in \mathscr{B}(\mathscr{H})$, where $\mathscr{H}$ is a Hilbert space, then [5] shows that $T$ is unitary, if $\|(T-\lambda) x\| \geq \operatorname{dist}(\lambda, \sigma(T))\|x\|$ for every $\lambda \in \rho(T)$ and every $x \in \mathscr{H}$. As shown in the proof of the next proposition, this inequality holds for TPN operators.

Proposition 4.7. If $\mathscr{H}$ is a Hilbert space and $T \in \mathscr{B}(\mathscr{H})$ is TPN and has real spectrum $\sigma(T)$ then $T$ is self-adjoint.

Proof. Since $T-\lambda$ is paranormal for every $\lambda \in \mathbf{C},(T-\lambda)^{-1}$ is paranormal for every $\lambda \in \rho(T)$ and hence $\left\|(T-\lambda)^{-1}\right\|=$ $r\left((T-\lambda)^{-1}\right)=\max \left\{|\mu-\lambda|^{-1} \mid \mu \in \sigma(T)\right\}=1 / \operatorname{dist}(\lambda, \sigma(T))$. Thus, for any $x \in \mathscr{H}$ and any $\lambda \in \rho(T)$ :

$$
\begin{aligned}
& \|x\| \operatorname{dist}(\lambda, \sigma(T))=\left\|(T-\lambda)^{-1}(T-\lambda) x\right\| \operatorname{dist}(\lambda, \sigma(T)) \\
& \quad \leq\left\|(T-\lambda)^{-1}\right\|\|(T-\lambda) x\| \operatorname{dist}(\lambda, \sigma(T))=\|(T-\lambda) x\| .
\end{aligned}
$$

Now apply [11].

Corollary 4.8. If $T$ is TPN then $X_{T}(\{\lambda\})=\operatorname{ker}(T-\lambda)$, for any $\lambda \in \mathbf{C}$.

Proof. It follows from Corollary 2.4 that if $T$ has SVEP then $x \in X_{T}(\{\lambda\})$ if and only if $\left\|(T-\lambda)^{n} x\right\|^{1 / n} \rightarrow 0$. If $T$ is TPN then $\left\|(T-\lambda)^{n} x\right\|^{1 / n} \geq\|(T-\lambda) x\|$. Hence $X_{T}(\{\lambda\}) \subseteq \operatorname{ker}(T-\lambda)$. Moreover, $\operatorname{ker}(T-\lambda) \subseteq X_{T}(\{\lambda\})$ (this is true in general, whether or not $T$ has SVEP and whether or not $X_{T}(\{\lambda\})$ is closed).

Corollary 4.9. If $T$ is TPN and $\lambda \in \sigma(T)$ is isolated, then $\lambda$ is an eigenvalue.

Proof. By [4, Proposition 1.3.10], if $\lambda$ is isolated in $\sigma(T)$ then $X=$ $X_{T}(\{\lambda\})+X_{T}(\sigma(T) \backslash\{\lambda\})$, and by Corollary 4.8, if $\operatorname{ker}(T-\lambda)=\{0\}$, then $X=X_{T}(\sigma(T) \backslash\{\lambda\})$. This contradicts $\lambda \in \sigma(T)$.

Proposition 4.10. Let $\mathscr{H}$ be a Hilbert space. The spectrum $\sigma(T)$ of a pure TPN operator $T \in \mathscr{B}(\mathscr{H})$ contains no isolated points.

Proof. By Corollary 4.9, every isolated point of $\sigma(T)$ is an eigenvalue of $T$. Suppose $T$ is non-zero and pure. If there is an isolated 
point, i.e., an eigenvalue $\lambda$ with $|\lambda|=\|T\|$, then Lemma 1.10 applies directly to give us a contradiction in the form of a reducing subspace on which the restriction of $T$ is normal. If all isolated points $\lambda \in \sigma(T)$ satisfy $|\lambda|<\|T\|$, choose any isolated point $\lambda \in \sigma(T)$ and then choose $\kappa \in \rho(T)$ so that $|\kappa-\lambda|<\operatorname{dist}(\kappa, \sigma(T) \backslash\{\lambda\})$. For notational simplicity we may suppose that $\kappa=0$, i.e., that $T$ is invertible. The inverse $T^{-1}$ will be paranormal as well as pure, as is easily seen. Moreover, since $\lambda$ is an eigenvalue of $T, 1 / \lambda$ is an eigenvalue of $T^{-1}$. Also, $|1 / \lambda|=r\left(T^{-1}\right)$, so that Lemma 1.10 may be applied (via Corollary 4.5): $\operatorname{ker}\left(T^{-1}-\lambda^{-1}\right)$ is reducing and $T^{-1} \mid \operatorname{ker}\left(T^{-1}-\lambda^{-1}\right)$ is normal. This contradicts the purity of $T^{-1}$.

COROllaRy 4.11 (cf. [14]). If $T \in \mathscr{B}(\mathscr{H})$ is TPN and has countable spectrum, then $T$ is normal.

Proof. It suffices to show that if $T$ is TPN and pure and has countable spectrum, then $T$ is zero. If $T$ is non-zero, then $\sigma(T)$ contains non-zero points (by Corollary 4.5). This contradicts Proposition 4.10.

We now show that $X_{T}(F)$ is closed when $F$ is closed.

LEMMA 4.12. Let $T$ be TPN on the Banach space $X$ and let $x \in X$ be a unit vector with local spectrum $\sigma_{T}(x)$ and local resolvent $\rho_{T}(x)$. For $n=1,2, \ldots$ let $x_{n}(\lambda)$ be the uniquely determined analytic function from $\rho_{T}(x)$ into $X_{T}\left(\sigma_{T}(x)\right)$ for which $(T-\lambda)^{n} x_{n}(\lambda)=x$ on $\rho_{T}(x)$. Then

$$
\left\|x_{1}(\lambda)\right\|^{n} \leq\left\|x_{n}(\lambda)\right\| \quad \text { for all } \lambda \in \rho_{T}(x) \text { and } n=1,2, \ldots
$$

Moreover, if $\lambda_{0} \in \rho_{T}(x) \backslash \Omega$, where $\Omega$ is an open neighborhood of $\sigma_{T}(x)$, and if $\Gamma \subseteq \Omega \backslash \sigma_{T}(x)$ is a finite disjoint union of simple closed rectifiable curves surrounding $\sigma_{T}(x)$ (i.e., the index $\operatorname{ind}_{\Gamma}(\lambda)=1$ for $\lambda \in \sigma_{T}(x)$ and $=0$ for $\left.\lambda \in \mathbf{C} \backslash \Omega\right)$ then

$$
x_{n}\left(\lambda_{0}\right)=-\frac{1}{2 \pi i} \int_{\Gamma} \frac{x_{1}(\lambda)}{\left(\lambda-\lambda_{0}\right)^{n}} d \lambda \quad \text { for } n=1,2, \ldots
$$

Proof. For the first part, by [3, Lemma 1.1.2] it is enough to show that $\left\|x_{n}(\lambda)\right\|^{2} \leq\left\|x_{n-1}(\lambda)\right\|\left\|x_{n+1}(\lambda)\right\|$ for any $\lambda \in \rho_{T}(x)$ and $n=$ $1,2, \ldots$ (Here $x_{0}(\lambda) \equiv x$.) These inequalities are immediate consequences of the TPN property:

$$
\begin{aligned}
\left\|x_{n}(\lambda)\right\|^{2} & =\left\|(T-\lambda) x_{n+1}(\lambda)\right\|^{2} \leq\left\|(T-\lambda)^{2} x_{n+1}(\lambda)\right\|\left\|x_{n+1}(\lambda)\right\| \\
& =\left\|x_{n-1}(\lambda)\right\|\left\|x_{n+1}(\lambda)\right\| .
\end{aligned}
$$


For $n \in \mathbf{N}$ define

$$
g_{n}\left(\lambda_{0}\right):=x_{n}\left(\lambda_{0}\right)+\frac{1}{2 \pi i} \int_{\Gamma} \frac{x_{1}(\lambda)}{\left(\lambda-\lambda_{0}\right)^{n}} d \lambda .
$$

As a consequence of Cauchy's theorem we obtain that

$$
\left(T-\lambda_{0}\right)^{n}\left(-\frac{1}{2 \pi i} \int_{\Gamma} \frac{x_{1}(\lambda)}{\left(\lambda-\lambda_{0}\right)^{n}} d \lambda\right)=x,
$$

and hence that $g_{n}\left(\lambda_{0}\right) \in \operatorname{ker}\left(T-\lambda_{0}\right)^{n}=\operatorname{ker}\left(T-\lambda_{0}\right)$, by Lemma 4.2. Since this is true for every point in some neighborhood of $\lambda_{0}$ and since $g_{n}$ is analytic in this neighborhood, it follows from SVEP that $g_{n}$ is zero. This completes the proof.

We can now give the estimate that is the main step.

LEMMA 4.13. If $T$ is TPN and if $x \in X$ is a unit vector with local resolvent $\rho_{T}(x)$ and local spectrum $\sigma_{T}(x)$ and if $(T-\lambda) x_{1}(\lambda)=x$ on $\rho_{T}(x)$ (where $x_{1}$ is analytic), then $\left\|x_{1}(\lambda)\right\| \leq\left(\operatorname{dist}\left(\lambda, \sigma_{T}(x)\right)\right)^{-1}$ for every $\lambda \in \rho_{T}(x)$.

Proof. As the proof of [3, Theorem 1.3.1], even though there the proof is given for Hilbert space only.

Proposition 4.14. Let $T$ be a TPN operator on the Banach space $X$ and let $F \subseteq \mathbf{C}$ be a closed set. Then $X_{T}(F)$ is closed.

Proof. It follows from Lemma 4.13 that if $\lambda \in \mathbf{C} \backslash F$, then $T-\lambda$ is bounded below on $X_{T}(F)$, hence has an invertible extension to $X_{T}(F)^{-}$. Consequently, $\sigma\left(T \mid X_{T}(F)^{-}\right) \subseteq F$ and hence $\sigma_{T}(x) \subseteq F$ for every $x \in X_{T}(F)^{-}$. Thus $X_{T}(F)^{-} \subseteq X_{T}(F)$.

For TPN operators in Hilbert space without point spectrum we can be even more specific, thus improving Corollary 4.3.

Proposition 4.15. Let $T \in \mathscr{B}(\mathscr{H})$ be a TPN operator with no eigenvalues and let $F$ be a closed set in $\mathbf{C}$. Then $E_{T}(F)=\mathscr{H}_{T}(F)=$ $\bigcap_{\lambda \notin F}(T-\lambda) \mathscr{H}$.

Proof. It will suffice to show that $\mathscr{H}_{T}(F) \supseteq \bigcap_{\lambda \notin F}(T-\lambda) \mathscr{H}$. So let $x$ belong to the set on the right and write $x=(T-\lambda) x(\lambda)$ for every $\lambda \notin$ $F$. Since $T$ has no eigenvalues, each $x(\lambda)$ is uniquely determined and consequently [3, Lemma 1.3.7], the function $\lambda \rightarrow\|x(\lambda)\|$ is lower semi-continuous on $\mathbf{C} \backslash F$. If $\sigma_{T}(x)$ is not a subset of $F$ then we can 
argue as on pp. 24-25 of [3] and obtain an open disc $D \subseteq C \backslash F$ with center contained in $\sigma_{T}(x)$ on which the function $x(\lambda)$ is bounded. It should be noted that this reasoning makes use of the estimate of Lemma 4.13, valid for points $\lambda_{0}$ outside $\sigma_{T}(x)$.

It remains to obtain a contradiction by showing that $x(\lambda)$ is analytic in $D$. Note first that by boundedness the function $x(\lambda)$ is weakly continuous; this also uses the assumption of no eigenvalues. Next, let $\lambda_{0} \in D$ and let $y_{0}=\left(T-\lambda_{0}\right)^{*} z_{0}$. For $\lambda \in D$

$$
\begin{aligned}
\langle x(\lambda) & \left., y_{0}\right\rangle-\left\langle x\left(\lambda_{0}\right), y_{0}\right\rangle \\
& =\left\langle(T-\lambda) x(\lambda)-\left(T-\lambda_{0}\right) x\left(\lambda_{0}\right)+\left(\lambda-\lambda_{0}\right) x(\lambda), z_{0}\right\rangle \\
& =\left(\lambda-\lambda_{0}\right)\left\langle x(\lambda), z_{0}\right\rangle
\end{aligned}
$$

which shows that the function $\lambda \rightarrow\left\langle x(\lambda), y_{0}\right\rangle$ is analytic on $D$ for every $y_{0} \in\left(T-\lambda_{0}\right)^{*} \mathscr{H}$, whenever $\lambda_{0} \in D$. Let $\Gamma$ denote a simple closed rectifiable curve in $D$ and let

$$
g:=\int_{\Gamma} x(\lambda) d \lambda
$$

taken in the weak sense. If $y_{0} \in\left(T-\lambda_{0}\right)^{*} \mathscr{H}$, then

$$
\left\langle g, y_{0}\right\rangle=\left\langle\int_{\Gamma} x(\lambda) d \lambda, y_{0}\right\rangle=\int_{\Gamma}\left\langle x(\lambda), y_{0}\right\rangle d \lambda=0 .
$$

Thus, since $\lambda_{0}$ is not an eigenvalue, $y_{0}$ may range through the dense subspace $\left(T-\lambda_{0}\right)^{*} \mathscr{H}$, hence $g=0$. If we now let $y_{0}$ be any vector in $\mathscr{H}$ then

$$
\int_{\Gamma}\left\langle x(\lambda), y_{0}\right\rangle d \lambda=\left\langle\int_{\Gamma} x(\lambda) d \lambda, y_{0}\right\rangle=\left\langle g, y_{0}\right\rangle=0,
$$

so that Morera's theorem tells us that $\left\langle x(\lambda), y_{0}\right\rangle$ is analytic on $D$. This shows that $x(\lambda)$ is analytic and contradicts the fact that $D$ contains points of $\sigma_{T}(x)$. Hence $\sigma_{T}(x) \subseteq F$ and the desired inclusion is proved.

5. An application. In Hilbert space, TPN operators without eigenvalues are what Neumann [10], calls admissible, i.e., $E_{T}(F)$ is closed whenever $F \subseteq \mathbf{C}$ is closed, so that the automatic continuity theory developed for admissible operators applies. This issue will be pursued in greater detail elsewhere, so suffice it here to mention a sample con- 
clusion, involving intertwining operators. This result is a consequence of [10, Corollary 3.3]. "Intertwines" means that $S \theta=\theta T$.

THEOREM. If $S \in \mathscr{B}(\mathscr{H})$ is TPN and has no eigenvalues then any linear transformation $\theta: X \rightarrow \mathscr{H}$ which intertwines a decomposable $T \in \mathscr{B}(X)$ with $S$ is automatically continuous.

In conclusion a few problems that come to mind naturally in the present context:

(a) Are TPN operators always admissible?

(b) Does a TPN operator have $(\beta)$ ? Probably the answer is yes.

(c) Is $E_{T}(\varnothing)=\{0\}$ when $T$ is TPN? If not for an arbitrary TPN operator $T$, is then $E_{T}(\varnothing)=\{0\}$ sufficient to ensure closedness of $E_{T}(F)$, i.e., admissibility and hence equality with $X_{T}(F)$ ?

\section{REFERENCES}

[1] T. Ando, Operators with a norm condition, Acta Sci. Math. Szeged, 33 (1972), 169-178.

[2] B. A. Barnes, Operators which satisfy polynomial growth conditions, Pacific J. Math., 138 (1989), 209-219.

[3] K. Clancey, Seminormal Operators, Lecture Notes in Math., vol. 742, Springer, Berlin-Heidelberg-New York, 1979.

[4] I. Colojoară and C. Foiaş, Theory of Generalized Spectral Operators, Gordon \& Breach, New York, 1968.

[5] W. F. Donaghue, Jr., On a problem of Nieminen, Inst. Hautes Études Sci. Publ. Math., 16 (1963), 127-129.

[6] R. G. Douglas, On majorization, factorization and range inclusion of operators on Hilbert space, Proc. Amer. Math. Soc., 17 (1966), 413-415.

[7] S. Frunză, A characterization of regular Banach algebras, Rev. Roumaine Math. Pures Appl., 18 (1973), 1057-1059.

[8] K. B. Laursen, Algebraic spectral subspaces and automatic continuity, Czechoslovak Math. J., 38 (113) (1988), 157-172.

[9] K. B. Laursen and P. Vrbová, Some remarks on the surjectivity spectrum of linear operators, Czechoslovak Math. J., 39 (114) (1989), 730-739.

[10] M. M. Neumann, Decomposable operators and generalized intertwining linear transformations, in : Operator Theory: Advances and Applications, vol. 28, Birkhäuser, Basel, 1988, pp. 209-222.

[11] T. Nieminen, $A$ condition for the selfadjointness of a linear operator, Ann. Acad. Sci. Fenn., Ser. A I (no. 316) (1962), 3-5.

[12] M. Putinar, Hyponormal operators are subscalar, J. Operator Theory, 12 (1984), 385-395.

[13] M. Radjabalipour, On majorization and normality of operators, Proc. Amer. Math. Soc., 62 (1977), 105-110.

[14] Chaoxing Qiu, Paranormal operators with countable spectrum are normal operators, J. Math. Res. Exposition, 7 (1987), 591-594. 
[15] J. Stampfli and B. Wadhwa, On dominant operators, Monatsh. Math., 84 (1977), 143-153.

[16] B. Sz.-Nagy and C. Foiaş, Harmonic Analysis of Operators on Hilbert Space, North-Holland, Amsterdam, 1970.

Received November 27, 1989 and in revised form July 2, 1990.

KøBENHAVNS UNIVERSITET

2100 COPENHAGen, DENMARK 


\title{
PACIFIC JOURNAL OF MATHEMATICS EDITORS
}

V. S. VARADARAJAN

(Managing Editor)

University of California

Los Angeles, CA 90024-1555-05

HeRbert Clemens

University of Utah

Salt Lake City, UT 84112

F. Michael Christ

University of California

Los Angeles, CA 90024-1555

THOMAS ENRIGHT

University of California, San Diego

La Jolla, CA 92093
Nicholas ERcolani

University of Arizona

Tucson, AZ 85721

R. FINN

Stanford University

Stanford, CA 94305

VAUGHAN F. R. JONES

University of California

Berkeley, CA 94720

Steven Kerckhoff

Stanford University

Stanford, CA 94305

\section{C. MOORE}

University of California

Berkeley, CA 94720

Martin ScharlemanN

University of California

Santa Barbara, CA 93106

HAROLD STARK

University of California, San Diego

La Jolla, CA 92093

\section{ASSOCIATE EDITORS}
R. Arens
E. F. BECKENBACH
B. H. NeumanN
F. WoLF
K. YoshidA
(1906-1982)
(1904-1989)

\section{SUPPORTING INSTITUTIONS}

UNIVERSITY OF ARIZONA

UNIVERSITY OF BRITISH COLUMBIA

CALIFORNIA INSTITUTE OF TECHNOLOGY

UNIVERSITY OF CALIFORNIA

MONTANA STATE UNIVERSITY

UNIVERSITY OF NEVADA, RENO

NEW MEXICO STATE UNIVERSITY

OREGON STATE UNIVERSITY

\author{
UNIVERSITY OF OREGON \\ UNIVERSITY OF SOUTHERN CALIFORNIA \\ STANFORD UNIVERSITY \\ UNIVERSITY OF HAWAII \\ UNIVERSITY OF TOKYO \\ UNIVERSITY OF UTAH \\ WASHINGTON STATE UNIVERSITY \\ UNIVERSITY OF WASHINGTON
}

The Supporting Institutions listed above contribute to the cost of publication of this Journal, but they are not owners or publishers and have no responsibility for its content or policies.

\begin{abstract}
Mathematical papers intended for publication in the Pacific Journal of Mathematics should be in typed form or offset-reproduced (not dittoed), double spaced with large margins. Please do not use built up fractions in the text of the manuscript. However, you may use them in the displayed equations. Underline Greek letters in red, German in green, and script in blue. The first paragraph must be capable of being used separately as a synopsis of the entire paper. In particular it should contain no bibliographic references. Please propose a heading for the odd numbered pages of less than 35 characters. Manuscripts, in triplicate, may be sent to any one of the editors. Please classify according to the 1991 Mathematics Subject Classification scheme which can be found in the December index volumes of Mathematical Reviews. Supply name and address of author to whom proofs should be sent. All other communications should be addressed to the managing editor, or Elaine Barth, University of California, Los Angeles, California 90024-1555-05.

There are page-charges associated with articles appearing in the Pacific Journal of Mathematics. These charges are expected to be paid by the author's University, Government Agency or Company. If the author or authors do not have access to such Institutional support these charges are waived. Single authors will receive 50 free reprints; joint authors will receive a total of 100 free reprints. Additional copies may be obtained at cost in multiples of 50 .
\end{abstract}

The Pacific Journal of Mathematics (ISSN 0030-8730) is published monthly except for July and August. Regular subscription rate: $\$ 190.00$ a year (10 issues). Special rate: $\$ 95.00$ a year to individual members of supporting institutions.

Subscriptions, orders for numbers issued in the last three calendar years, and changes of address should be sent to Pacific Journal of Mathematics, P.O. Box 969, Carmel Valley, CA 93924, U.S.A. Old back numbers obtainable from Kraus Periodicals Co., Route 100, Millwood, NY 10546.

The Pacific Journal of Mathematics at P.O. Box 969, Carmel Valley, CA 93924 (ISSN 0030-8730) is published monthly except for July and August. Second-class postage paid at Carmel Valley, California 93924, and additional mailing offices. Postmaster: send address changes to Pacific Journal of Mathematics, P.O. Box 969, Carmel Valley, CA 93924.

PUBLISHED BY PACIFIC JOURNAL OF MATHEMATICS, A NON-PROFIT CORPORATION Copyright (C) 1992 by Pacific Journal of Mathematics 


\section{Pacific Journal of Mathematics}

Vol. 152, No. $2 \quad$ February, 1992

Edoardo Ballico, On the restrictions of the tangent bundle of the

Grassmannians

Edward Burger, Homogeneous Diophantine approximation in

$S$-integers

Jan Dijkstra, Jan van Mill and Jerzy Mogilski, The space of

infinite-dimensional compacta and other topological copies of $\left(l_{f}^{2}\right)^{\omega} \ldots 255$

Mike Hoffman, Multiple harmonic series ..................... 275

Wu Hsiung Huang, Superharmonicity of curvatures for surfaces of constant

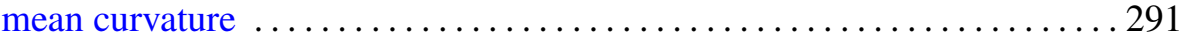

George Kempf, Pulling back bundles ......................... 319

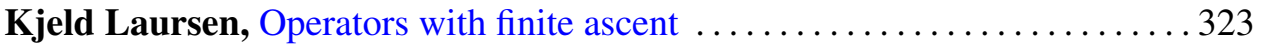

Andrew Solomon Lipson, Some more states models for link invariants . . . . 337

Xiang Yang Liu, Bloch functions of several complex variables .......... 347

Madabusi Santanam Raghunathan, A note on generators for arithmetic

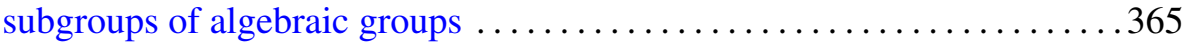

Marko Tadić, Notes on representations of non-Archimedean $\operatorname{SL}(n) \ldots \ldots 375$ 